\title{
A Novel Frequency Scanning Leaky-Wave Antenna Based on Corrugated Substrate Integrated Waveguide
}

\author{
Ke Chen $\mathbb{D}^{1},{ }^{1}$ Yunhua Zhang $\mathbb{D}^{1},{ }^{1}$ Siyuan He, ${ }^{1}$ Haitao Chen, ${ }^{2}$ and Guoqiang Zhu ${ }^{1}$ \\ ${ }^{1}$ School of Electronic Information, Wuhan University, Wuhan 430072, China \\ ${ }^{2}$ Wuhan Maritime Communication Research Institute, Wuhan 430079, China
}

Correspondence should be addressed to Ke Chen; 2013202120030@whu.edu.cn and Yunhua Zhang; zhangyunhua@whu.edu.cn

Received 17 April 2018; Revised 1 July 2018; Accepted 24 July 2018; Published 18 September 2018

Academic Editor: Yu Jian Cheng

Copyright ( $92018 \mathrm{Ke}$ Chen et al. This is an open access article distributed under the Creative Commons Attribution License, which permits unrestricted use, distribution, and reproduction in any medium, provided the original work is properly cited.

\begin{abstract}
In order to facilitate the integration of active devices, a leaky-wave antenna (LWA) based on corrugated substrate integrated waveguide (CSIW) structure is proposed. By introducing rectangular ring slots on the CSIW structure, the proposed LWA is capable of beam scanning from backward to forward. The rectangular ring slots do not only enable energy leaking but also provide series capacitance. In addition, the grounded vias and quarter-wave open-circuit stubs provide shunt inductance. Thus, the proposed CSIW exhibits left-handed characteristic which enables beam scanning through the broadside. To further facilitate the design and suppress open-stopband of beam scanning antenna based on CSIW structure, an equivalent circuit model is developed and the relationships between structure parameters and the equivalent circuit parameters are presented. Based on this design guide, a leaky wave antenna is designed with radiation angle ranging from $-53^{\circ}$ to $23^{\circ}$. This proposed novel CSIW LWA has great potential in tunable or reconfigurable antenna design due to its ease of integration with active devices.
\end{abstract}

\section{Introduction}

Leaky-wave antenna is widely used in wireless communication systems in recent years, due to their low-profile, high directivity, and frequency-scanning capability [1]. Leakywave antenna was firstly designed by loading rectangular waveguide with periodically distributed slots, which allowed for energy leaking [1]. The rectangular waveguide based LWAs can provide high gain, but the beam scanning from forward to backward direction suffered a prohibition at the boresight due to the open-stopband. This limitation was removed by designing LWAs based on composite right/lefthanded (CRLH) transmission line [2], which can support continuous beam scanning from backward to forward.

More recently, with the development of substrate integrated waveguide (SIW), various LWAs based on SIW structure are designed due to the advantages of low loss, low profile, and ease of fabrication and integration. SIW LWAs with periodically arranged transverse slots were demonstrated to achieve high directivity and low sidelobe level $[3,4]$. And LWAs array based on SIW structure was proposed to obtain high efficiency $[5,6]$. Furthermore, to realize beam scanning from backward to forward, SIW LWAs were designed by periodically loading of transversal and longitudinal slots [7], interdigital slots [8, 9]. However, it is challenging for the conventional SIW structure to be integrated with the active device. Since it has metallic vias to ground, it is difficult to design DC bias on SIW structure. Recently, the emerging of corrugated SIW (CSIW) exhibits great potentials in integrating with active circuits. In CSIW structure, the quarter-wavelength microstrip stubs, instead of metallic vias, are employed to realize electric side wall [10]. Therefore, the top and bottom conductors are isolated at DC and this structure has the ability to ease implementation of DC bias for active circuits. However, the bandwidth of CSIW structure is narrower than SIW structure because the length of microstrip open-circuit stub is a quarter wavelength at the band center frequency. Therefore, the designing of frequency scanning LWA on CSIW structure is more complex than SIW structure.

In this paper, a method for designing LWA capable of beam scanning from backward to forward base on CSIW 


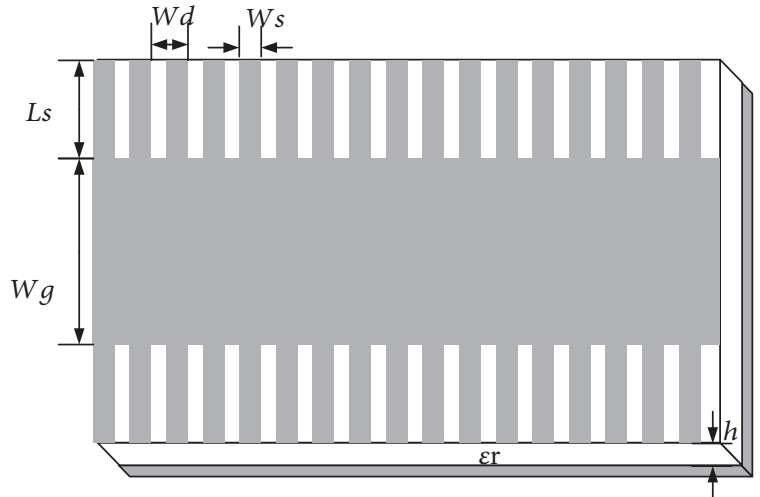

(a)

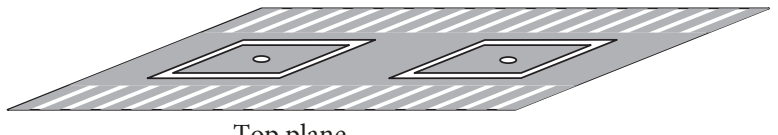

Top plane

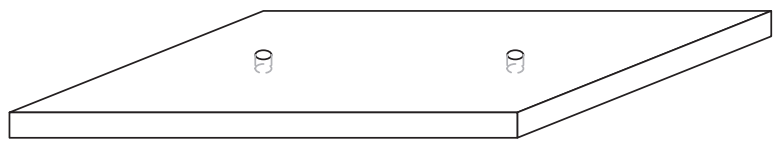

Substrate

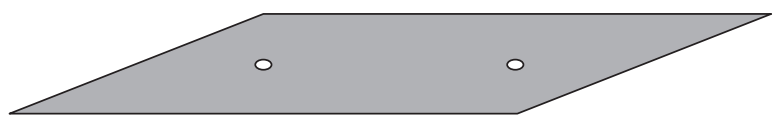

Bottom plane

(c)

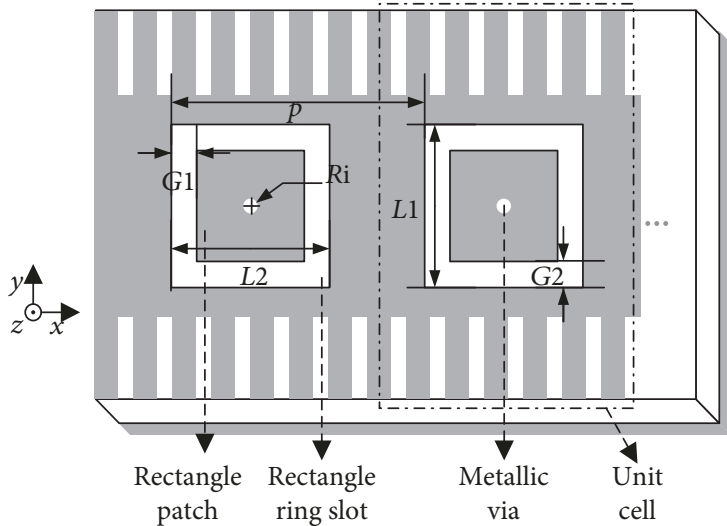

(b)

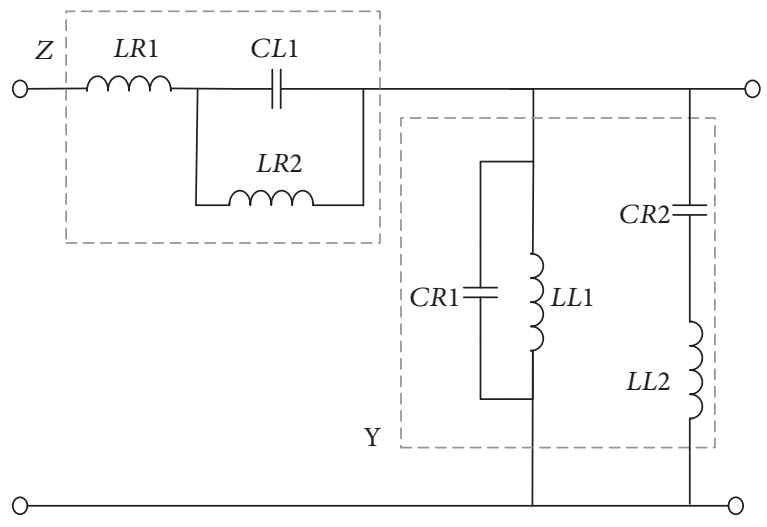

(d)

FIgure 1: (a) Structure of CSIW. (b) Top view of CSIW-CRLH structure. (c) Exploded view of CSIW-CRLH structure. (d) Equivalent circuit of unit cell CSIW-CRLH structure. $L_{s}=7 \mathrm{~mm}, W_{g}=7 \mathrm{~mm}, W_{d}=1.4 \mathrm{~mm}, W_{s}=0.7 \mathrm{~mm}, h=1 \mathrm{~mm}, \varepsilon_{r}=3.55$.

structure is presented. By introducing rectangular ring slots on the CSIW structure, the proposed structure exhibits CRLH transmission property, while the slots enable energy leaking and beam scanning. The proposed LWA is advantageous in simplifying the integration with active devices, owing to its isolated equipotential plane on single layer PCB structure. In addition, a design guide is presented for balanced unit cell structure design and the subsequent open-stopband suppressing. At last, a LWA based on CSIW-CRLH structure is designed, fabricated, and measured, and the design method is well validated by the measured results.

This paper is organized as follows. In Section 2, the analysis and design method of CSIW-CRLH unit cell structure is presented. In Section 3, a balanced CSIW-CRLH LWA with low cross-polarization is designed, and the experimental results are presented. Finally, a conclusion is drawn in Section 4.

\section{Unit Cell Structure and Design Guide}

The CSIW confines energy transmission in the same manner as that of the SIW. As a contrast, quarter-wavelength microstrip stubs are employed to form electric side wall, instead of using metallic vias as in the conventional SIW. The schematic of a CSIW structure is shown in Figure 1(a). It shows that the CSIW structure has two isolated DC equipotential planes including the top plane and the bottom plane, which provide a foundation for implementing DC bias. Nevertheless, the two isolated DC equipotential planes are not on the same layer, so it is still not straightforward to implement the DC bias.

Figures 1(b) and 1(c) show the geometry of the proposed CSIW-CRLH structure. By using the rectangular slots implemented on the top plane, three isolated DC equipotential planes are obtained, including the rectangular patch on the top plane, the rest metallic on the top plane, and the bottom ground plane. Furthermore, to facilitate the implementation of DC bias, metallic vias are introduced to connect the central of rectangular patches to the bottom plane, such that two isolated DC equipotential planes can be realized on the same layer. Besides, the metallic vias to ground contribute to shunt inductance, and the rectangular ring slots on top of the CSIW provide series capacitance. These two parts are combined to form a composite right hand and left-hand property, namely, CSIW-CRLH. Moreover, the rectangular ring slots also enable energy leaking and beam scanning.

The equivalent circuit model of CSIW-CRLH unit cell structure is derived as shown in Figure 1(d), which consists of series impedance $Z$ and shunt admittance $Y$. And the propagation constant can be calculated as follows [2]:

$$
\beta p=\cos ^{-1}(1+Z Y)
$$




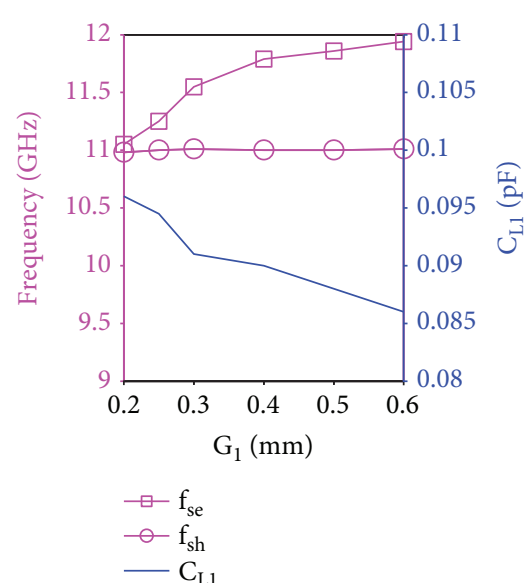

(a)

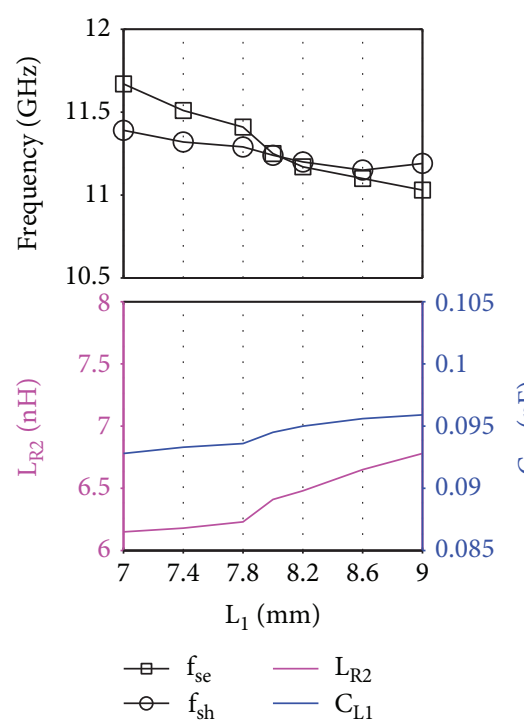

(c)

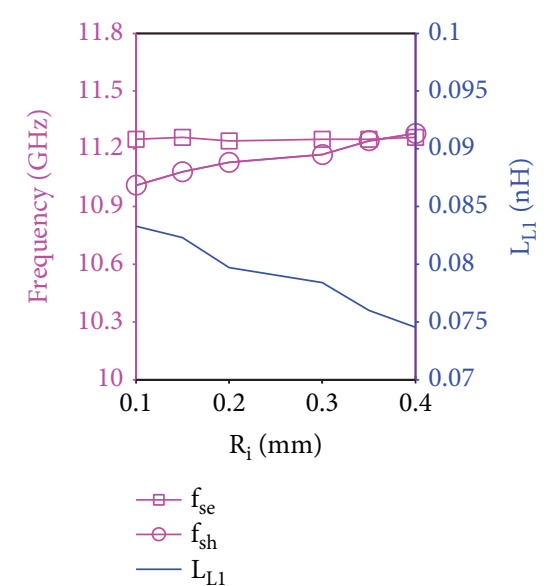

(b)

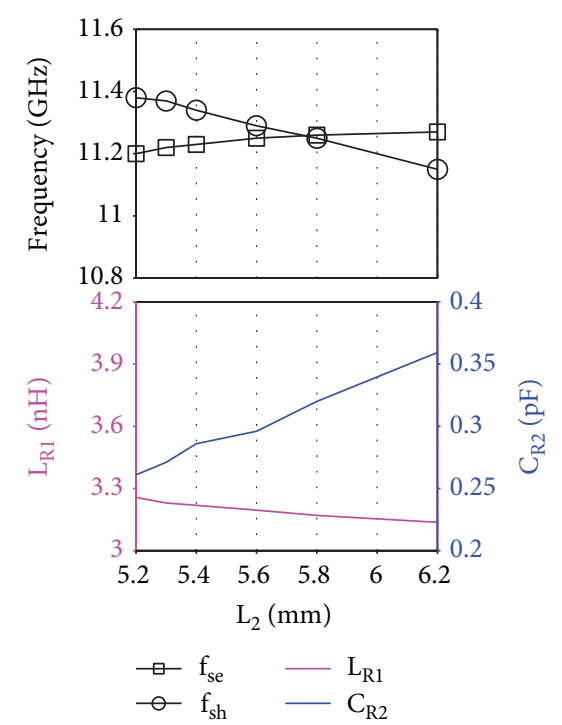

(d)

FIgURE 2: The impact of structure parameters on the equivalent circuit parameters, (a) $G_{1}$, the width of the transverse slots; (b) $R_{i}$, the radius of via; (c) $L_{1}$, length of transverse slots; (d) $L_{2}$, length of longitudinal slots. The initial structure parameters are listed in Table 1 , and then $f_{\text {se }}$ and $f_{\text {sh }}$ is $11.79 \mathrm{GHz}$ and $10.99 \mathrm{GHz}$, respectively.

Furthermore, the series impedance $Z$ consists of series capacitance $C_{L 1}$, series inductor $L_{R 1}$ (series with $C_{L 1}$ ) and $L_{R 2}$ (shunt with $C_{L 1}$ ), and the shunt admittance $Y$ consists of main shunt capacitance $C_{R 1}$, main shunt inductor $L_{L 1}$, shunt capacitance $C_{R 2}$, and shunt inductor $L_{L 2}$. Omitting the $L_{L 2}$ does not have a major impact on the calculation of zero points of $Y$, so the series impedance $Z$ and shunt admittance $Y$ is obtained:

$$
\begin{aligned}
Z & =j \omega L_{R 1} \frac{\omega^{2}-\omega_{s e 0}^{2}}{\omega^{2}-\omega_{s e 1}^{2}}, Y \approx-j \frac{C_{R 1}}{\omega} \frac{\left(\omega^{2}-\omega_{s h 0}^{2}\right)}{\left(\omega^{2}-\omega_{s h 1}^{2}\right)}, \\
\omega_{s e 0} & =\frac{1}{C_{L 1}}\left(\frac{1}{L_{R 1}}+\frac{1}{L_{R 2}}\right), \omega_{s e 1}=\frac{1}{C_{L 1} L_{R 2}}, \\
\omega_{s h 0} & =\frac{1}{L_{L 1}\left(C_{R 1}+C_{R 2}\right)}, \omega_{s h 1}=\frac{1}{L_{L 2} C_{R 2}},
\end{aligned}
$$

where $\omega_{s e 0}$ represents the primary zero point of $Z$ and $\omega_{s h}$ represents the primary zero point of $Y$. In addition, $\omega_{s e 1}$ and $\omega_{s h 1}$ are the pole point of $Z$ and $Y$, respectively, and they are far away from the balanced frequency in general.

The stop-band of CRLH structure will be suppressed when $\omega_{\text {se0 }}=\omega_{\text {sh } 0}$ [2]. In order to design the CSIW-CRLH structure without stop-band expediently, the relationships between the structure parameters and the equivalent circuit parameters are analyzed.

The relationships between the structure parameters and the equivalent circuit parameters are shown in Figure 2, and $f_{s e}=\omega_{\text {seo }} / 2 \pi$ and $f_{s h}=\omega_{s h 0} / 2 \pi$. As shown in Figure $2(\mathrm{a}), G_{1}$ has a large impact on $f_{\text {se }}$, and when $G_{1}$ decreases from $0.4 \mathrm{~mm}$ to $0.25 \mathrm{~mm}, C_{L 1}$ increases from $0.089 \mathrm{pF}$ to $0.0945 \mathrm{pF}$, which leads series resonant frequency $f_{\text {se }}$ to decrease from $11.79 \mathrm{GHz}$ to $11.25 \mathrm{GHz}$. Then, from Figure 2(b), by increasing $R_{i}$ from $0.1 \mathrm{~mm}$ to $0.35 \mathrm{~mm}, L_{L 1}$ decreases from $0.083 \mathrm{pF}$ to $0.076 \mathrm{pF}$, which leads shunt 
TABLE 1: Structure parameter of CSIW-CRLH.

\begin{tabular}{lcc}
\hline Parameter & Initial design $(\mathrm{mm})$ & Final design $(\mathrm{mm})$ \\
\hline$G_{1}$ & 0.4 & 0.25 \\
$L_{1}$ & 8 & 8 \\
$G_{2}$ & 0.2 & 0.2 \\
$L_{2}$ & 5.8 & 5.8 \\
$p$ & 12.6 & 12.6 \\
$R_{i}$ & 0.1 & 0.35 \\
\hline
\end{tabular}

resonant frequency $f_{s h}$ to increase from $10.99 \mathrm{GHz}$ to $11.25 \mathrm{GHz}$. So the $f_{s e}$ and $f_{\text {sh }}$ reach the target frequency, and the stop-band has been suppressed.

In addition, the relationship between $L_{1}$ and $f_{s e}, f_{s h}$ becomes more complex for $L_{1}$ has an impact on several circuit parameters. From Figure 2(c), $L_{1}$ has a large impact on $f_{s e}$ and a slight impact on $f_{\text {sh }}$. Similarly, $L_{2}$ has a little impact on $f_{s e}$ and a bit impact on $f_{s h}$, as shown in Figure 2(d).

By changing $G_{1}$ and $L_{1}$, the value of $C_{L 1}$ and $L_{R 2}$ can be adjusted, which will determine the value of $f_{s e}$. Meanwhile, the variation of $L_{1}$ will result in a change of $f_{s h}$. The increase of $R_{i}$, which decreases the value of $L_{L 1}$, will increase $f_{s h}$. Likewise, there is a negative correlation between $f_{\text {sh }}$ and $L_{2}$. Based on this analysis, the CSIW-CRLH structure without stopband can be designed conveniently.

Then the final structure parameters of CSIW-CRLH are obtained and listed in Table 1. Based on the structure parameters, the dispersion characteristics of the unit cell CSIWCRLH structure are simulated, thereafter the equivalent circuit parameters are extracted and the dispersion characteristic is predicted according to (1), as shown in Figure 3. The dispersion characteristics predicted by the circuit model are in good agreement with the simulated result, moreover, the stop-band of CRLH structure has been suppressed and $f_{s e}=f_{s h}=11.25 \mathrm{GHz}$. For the frequency band below the balanced frequency $11.25 \mathrm{GHz}$, the phase propagation is backward and it is termed as a left-hand region, while the frequency band above $11.25 \mathrm{GHz}$ is the right-hand region. This result indicates that the proposed CSIW structure has the ability of continuous beam scanning from backward to forward, thanks to the left-hand characteristic enabled by the rectangular ring slots.

\section{Simulation and Measurement}

For verification, a CSIW-CRLH LWA cascaded by ten unit cells is fabricated, as shown in Figure 4. A tapered microstrip line is employed to feed the proposed antenna. The prototype is fabricated with Rogers $4003 c\left(\varepsilon_{r}=3.55\right)$ substrate. The whole size of the fabricated LWA is $140 \mathrm{~mm} \times$ $28 \mathrm{~mm} \times 1 \mathrm{~mm}$.

The comparison of S-parameters obtained from measurement and simulation are shown in Figure 5. According to the results, the return loss curves from simulation and measurement are in close coincidence both in shape and trend. The measured return loss is better than $-10 \mathrm{~dB}$ from 10.2 $\mathrm{GHz}$ to $12.1 \mathrm{GHz}$, whereas the measured insertion loss

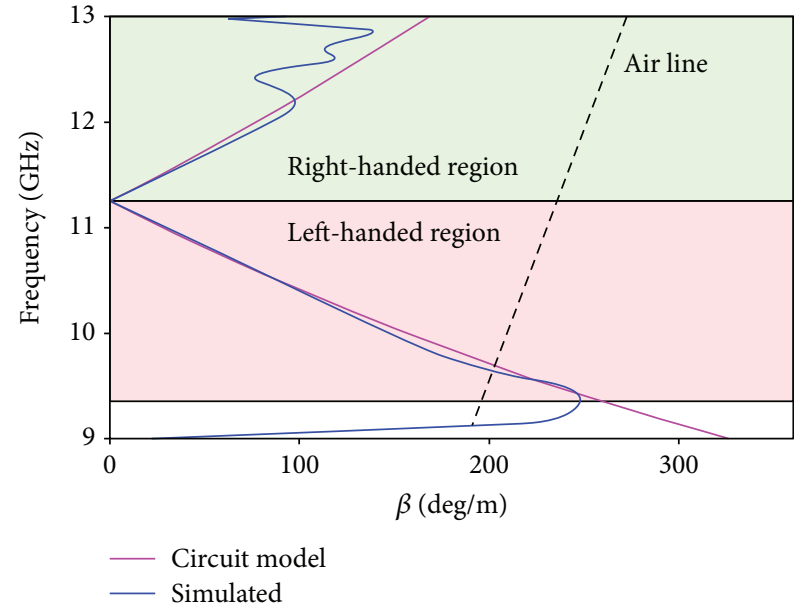

Figure 3: Comparison of the dispersion characteristics of the designed CSIW-CRLH obtained by full-wave simulation and equivalent circuit prediction. $L_{R 1}=3.17 \mathrm{nH}, L_{R 2}=6.41 \mathrm{nH}$, $C_{L 1}=0.0945 \mathrm{pF}, \quad L_{L 1}=0.076 \mathrm{nH}, \quad L_{L 2}=0.023 \mathrm{nH}, \quad C_{R 1}=2.21 \mathrm{pF}$, $C_{R 2}=0.32 \mathrm{pF}$.

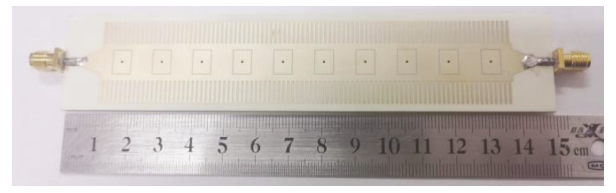

Figure 4: Photograph of the fabricated CSIW-CRLH LWA.

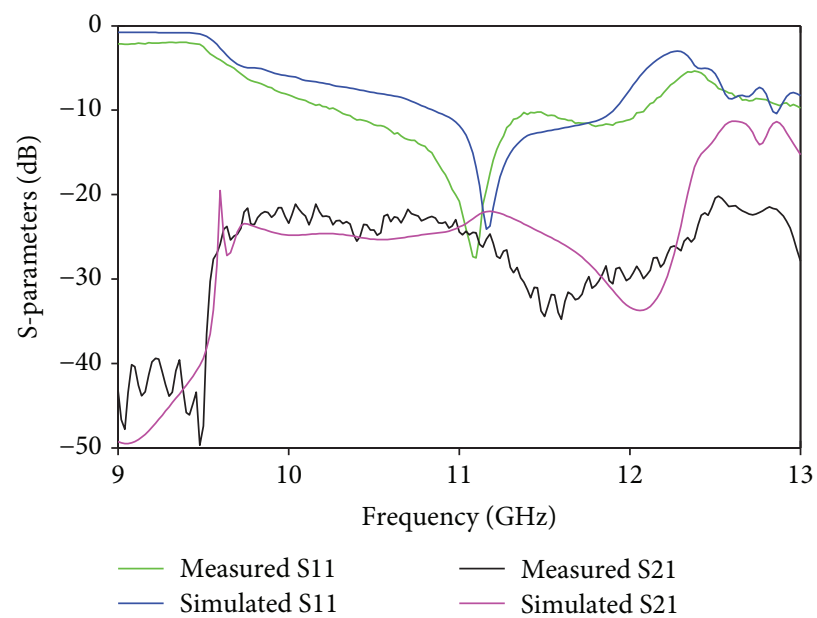

FIgURE 5: S-parameters of the proposed antenna.

is below $-20 \mathrm{~dB}$ in this range, which indicates that most of the energy are radiated.

The measured and simulated results of radiation angle and antenna gain are shown in Figure 6. It is shown that the radiation angles from measurement are in good agreement with that from the simulation. The measured results demonstrate that the beam scanning at least from $-53^{\circ}$ to $23^{\circ}$ as the frequency increases from $9.8 \mathrm{GHz}$ to $12 \mathrm{GHz}$ with broadside radiation at $11.2 \mathrm{GHz}$. Meanwhile, with the 


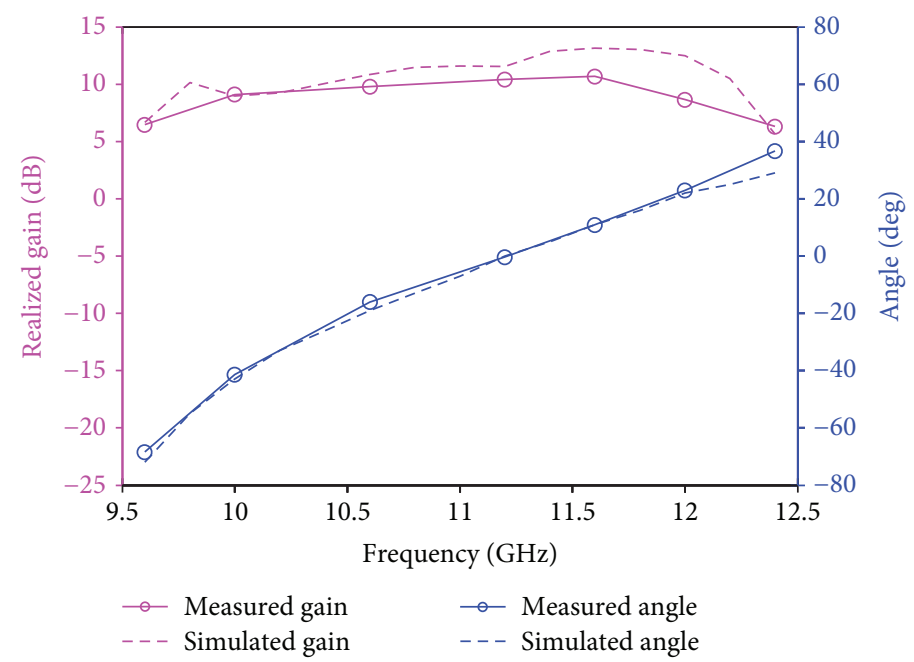

Figure 6: Beam angle and realized gain of the antenna.

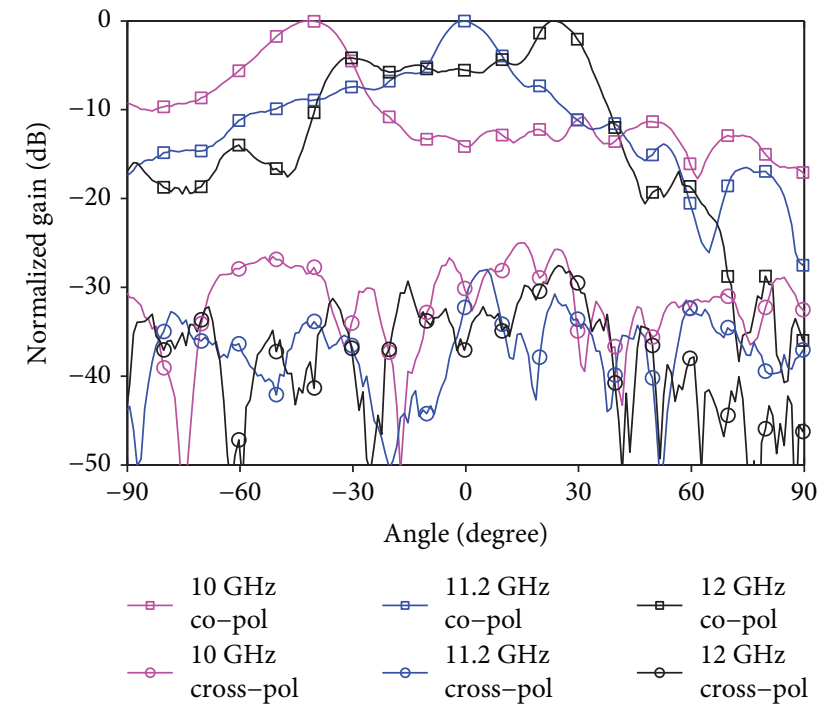

Figure 7: Measured radiation patterns of copolarization (above) and cross-polarization (below) in the E-plane of the fabricated LWA.

frequency ranging from $9.8 \mathrm{GHz}$ to $12 \mathrm{GHz}$, the gain is better than $8.5 \mathrm{~dB}$ and the maximum gain is $10.7 \mathrm{~dB}$ at $11.6 \mathrm{GHz}$. The measured results of antenna gain are about $0.5-1.5 \mathrm{~dB}$ lower than the simulated results in some regions, which is due to the dimensional tolerance and the material loss.

The normalized radiation patterns from measurement at $10 \mathrm{GHz}$ (backward radiation), $11.2 \mathrm{GHz}$ (broadside radiation), and $12 \mathrm{GHz}$ (forward radiation) of the fabricated LWA are shown in Figure 7. It can be seen that the crosspolarization levels are less than $-26 \mathrm{~dB}$ across the entire radiation region, and this is due to the far-field electric field cancellation of the symmetric longitudinal slots.

\section{Conclusion}

In this article, a novel LWA based on CSIW structure has been designed and demonstrated to support continuous beam scanning from backward to forward directions without an open-stopband at the boresight. The rectangular ring slots introduced in the CSIW play a vital role in the LWA design. First of all, the ring slots and the resulting patches contribute to series capacitance and shunt inductance, respectively, which lead to left-hand transmission property and backward beamforming. And this contribution has been studied by using a simple equivalent circuit model, which ultimately facilitate the design of the balanced CSIW-CRLH structure. Moreover, the slots enable energy leaking and beam scanning and it also brings about low cross-polarization in the E-plane. Lastly and most importantly, the introduced slots in CSIWCRLH facilitate the implementation of DC bias, thanks to the two isolated DC equipotential planes formed by the slots. This structure has great potential for application in the tunable circuit and reconfigurable antennas.

\section{Data Availability}

The dimension parameters of proposed structure data used to support the findings of this study are included in the article. The dimension parameters of the proposed structure are presented in Figure 1 and Table 1.

\section{Conflicts of Interest}

The authors declare that they have no conflicts of interest.

\section{Acknowledgments}

This work was supported by the National Natural Science Foundation of China (Grant no. 61301061 and Grant no. 61571333) and Wuhan Maritime Communication Research Institute.

\section{References}

[1] D. R. Jackson and A. A. Oliner, Leaky-Wave Antennas, John Wiley \& Sons, 2007. 
[2] C. Caloz and T. Itoh, Electromagnetic Metamaterials: Transmission Line Theory and Microwave Applications: The Engineering Approach, John Wiley \& Sons, 2005.

[3] M. M. Zhou, Y. J. Cheng, and W. N. Huang, "Substrate integrated slot array antenna with required radiation pattern envelope," International Journal of Antennas and Propagation, vol. 2016, Article ID 4031029, 4 pages, 2016.

[4] J. Liu, D. R. Jackson, Y. Li, C. Zhang, and Y. Long, "Investigations of SIW leaky-wave antenna for endfire-radiation with narrow beam and sidelobe suppression," IEEE Transactions on Antennas and Propagation, vol. 62, no. 9, pp. 4489-4497, 2014.

[5] Y. Geng, J. Wang, Y. Li, Z. Li, M. Chen, and Z. Zhang, "Highefficiency leaky-wave antenna array with sidelobe suppression and multibeam generation," IEEE Antennas and Wireless Propagation Letters, vol. 16, pp. 2787-2790, 2017.

[6] Y. Geng, J. Wang, Y. Li, Z. Li, M. Chen, and Z. Zhang, "Leakywave antenna array with a power-recycling feeding network for radiation efficiency improvement," IEEE Transactions on Antennas and Propagation, vol. 65, no. 5, pp. 2689-2694, 2017.

[7] Y.-L. Lyu, X. X. Liu, P. Y. Wang et al., "Leaky-wave antennas based on noncutoff substrate integrated waveguide supporting beam scanning from backward to forward," IEEE Transactions on Antennas and Propagation, vol. 64, no. 6, pp. 2155-2164, 2016.

[8] Y. D. Dong and T. Itoh, "Composite right/left-handed substrate integrated waveguide leaky-wave antennas," in Proceedings of the 39th European Microwave Conference, 2009, EuMC 2009, pp. 276-279, Rome, Italy, September 2009.

[9] C.-a. Wang and B. Hu, "Leaky-wave antenna with high gain and wide beam-scanning angle range based on novel SIWCRLH transmission line," International Journal of RF and Microwave Computer-Aided Engineering, vol. 26, no. 1, pp. 36-41, 2016.

[10] K. W. Eccleston, "Mode analysis of the corrugated substrate integrated waveguide," IEEE Transactions on Microwave Theory and Techniques, vol. 60, no. 10, pp. 3004-3012, 2012. 


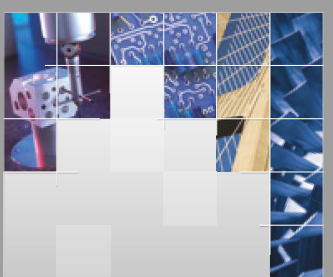

\section{Enfincering}
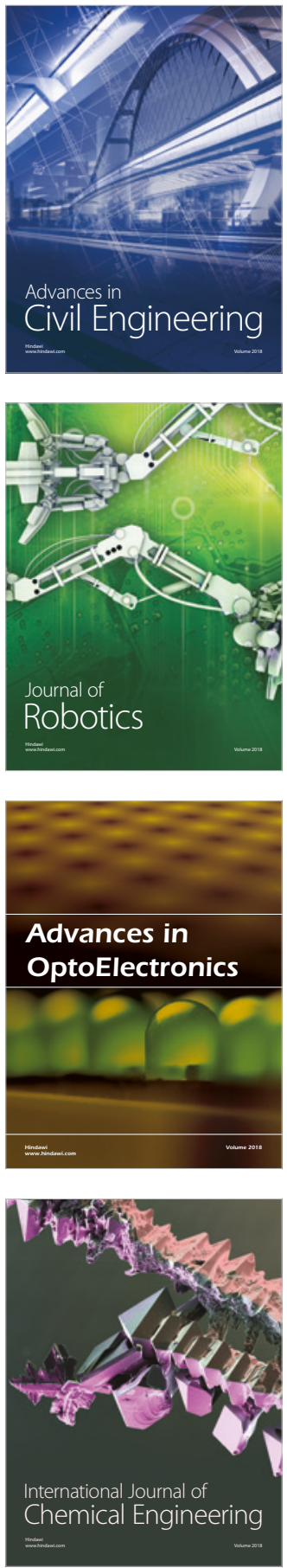

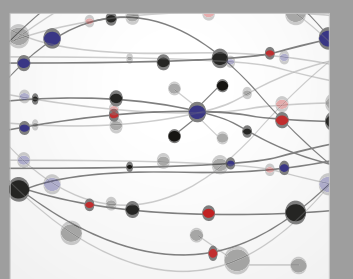

\section{Rotating \\ Machinery}

The Scientific World Journal

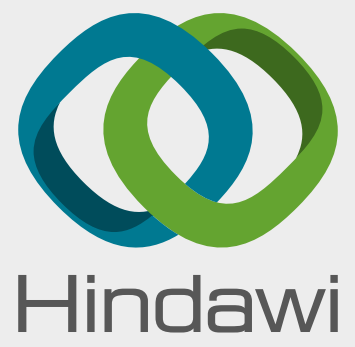

Submit your manuscripts at

www.hindawi.com
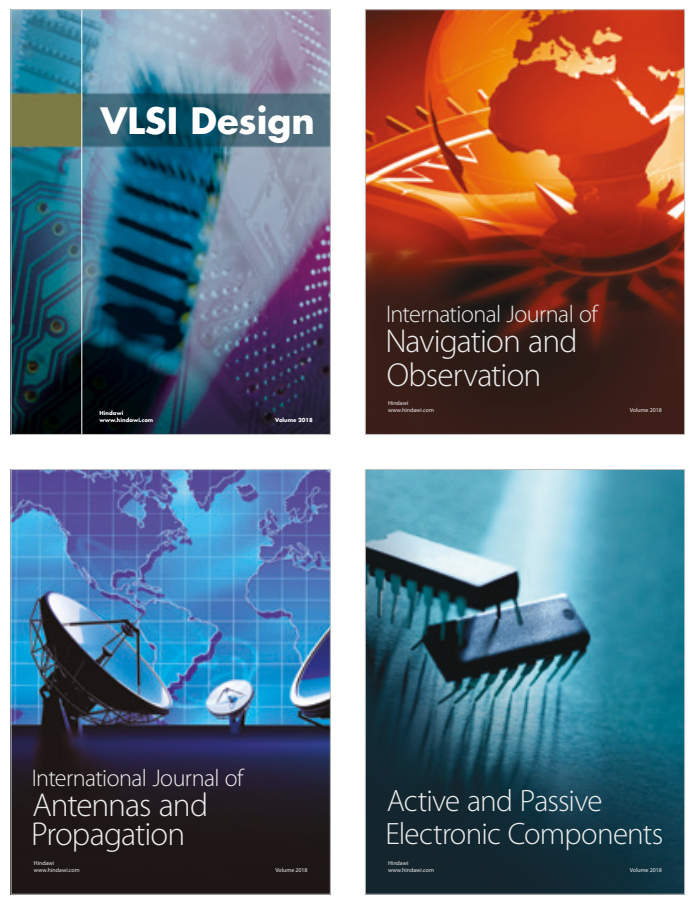
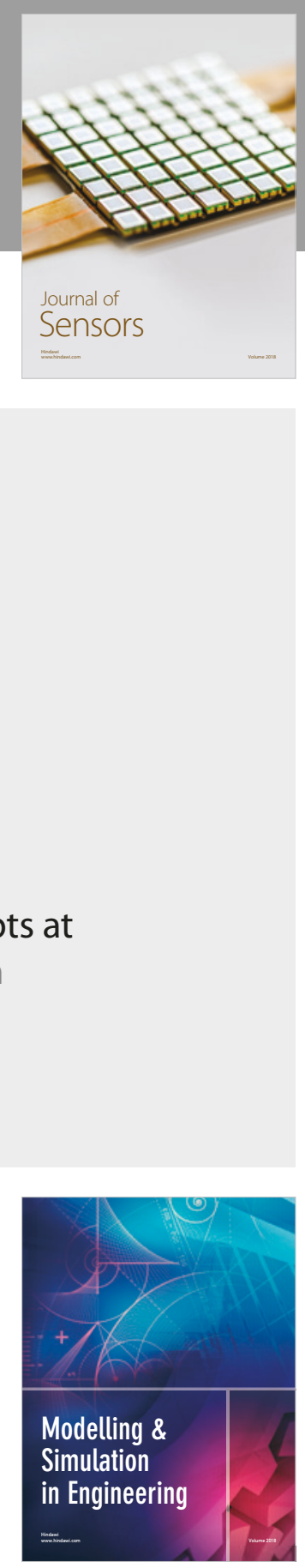

\section{Advances \\ Multimedia}
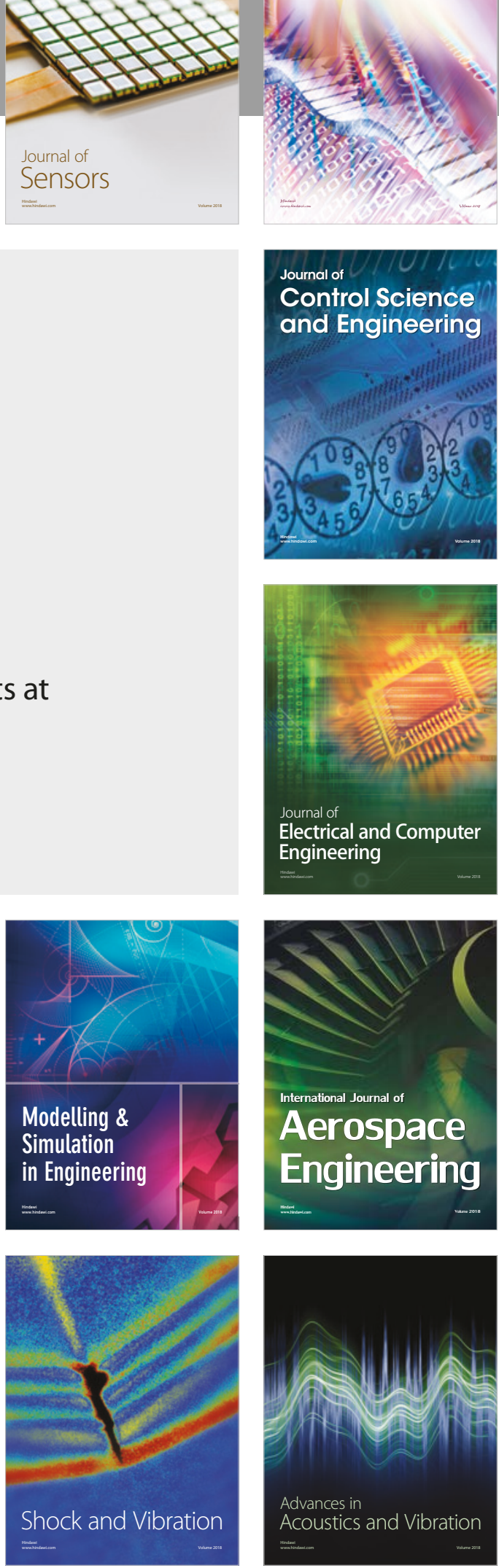